\title{
Identification of Oncosuppressing Effects of Seven Selenoproteins in Thyroid Cancer: Implications for Distinct Roles of Selenoproteins in Tumorigenesis
}

\section{Yang Zhao}

The First Affiliated Hospital of Xi'an Jiaotong University

\section{Pu Chen}

The First Affiliated Hospital of Xi'an Jiaotong University

Hong-jun Lv

The First Affiliated Hospital of Xi'an Jiaotong University

\section{Yuan Wu}

The First Affiliated Hospital of Xi'an Jiaotong University

Shu Liu

The First Affiliated Hospital of Xi'an Jiaotong University

\section{Xueyang Deng}

China Pharmaceutical University

Jiao Fu ( $\nabla$ fujiaoxyz@hotmail.com )

The First Affiliated Hospital of Xi'an Jiaotong University https://orcid.org/0000-0002-4970-4987

\section{Research Article}

Keywords: Selenoproteins, Thyroid cancer, Pathogenesis, Biological processes, Multiomic data mining study

Posted Date: April 13th, 2021

DOI: https://doi.org/10.21203/rs.3.rs-402154/v1

License: (c) (1) This work is licensed under a Creative Commons Attribution 4.0 International License. Read Full License 


\section{Abstract}

Background: Low selenium levels are associated with increased incidence and advanced stage of thyroid cancers. In response to changes in selenium levels, a hierarchy of selenoprotein biosynthesis allows tissue-specific fine-tuning of the 25 selenoproteins. To determine the role of individual selenoproteins on thyroid carcinogenesis, we carried out a multiomic data mining study.

Methods: The expressions of individual selenoproteins and their correlation with prognosis in thyroid cancers were analyzed using Oncomine, GEPIA and Kaplan-Meier plotter platforms. Co-expression analysis using cBioportal database was carried out to identify genes that are correlated with selenoproteins. GO and KEGG enrichment were further performed for genes correlated with selenoproteins that are clinically significant.

Results: DI01, GPX3, SELENOP, SELENOM SELENOS, SELENOO and SELENOV were significantly downregulated and with a poor prognosis in thyroid cancers. 35 biological processes including $\mathrm{GO}$ : 0045926 (negative regulation of growth) and 23 biological processes including G0:0001525 (angiogenesis) and GO:0007155 (cell adhesion) were enriched in DI01-positively and DI01-negatively correlated genes, respectively. 41 biological processes including GO: 0045926 (negative regulation of growth) and 23 biological processes including G0:0007165 (signal transduction) and G0: 0000165 (MAPK cascade) were enriched in GPX3-positively and GPX3-negatively correlated genes, respectively. The antitumor effects of SELENOM and SELENOS might be attributed to their protection against endoplasmic reticulum (ER) stress. SELENOO was revealed to be correlated with ER stress, mitochondria translation and telomere maintenance. Biological processes of SELENOV-correlated genes were enriched in oxidation-reduction process and ER calcium ion homeostasis. Moreover, cell adhesion and angiogenesis were also shown to be negatively regulated by SELENOV, providing an anti-metastatic effect similar as DI01.

Conclusion: This study confirms the distinct roles of the 25 selenoproteins in thyroid cancer pathogenesis, providing useful information to uncover the currently unknown functions of selenoproteins and open up the possibility for targeted regulation of individual selenoproteins for treatment of thyroid cancer.

\section{Background}

Thyroid nodules are the most common thyroid disease with a malignant rate of $7-15 \%{ }^{[1]}$. The incidence of thyroid cancer has been linked to low body selenium levels ${ }^{2{ }^{2]}}$. Selenium is an essential trace element exerting its biological activity through the incorporation of selenocysteine (Sec) into a unique class of proteins termed selenoproteins. In humans, there are 25 selenoproteins, whose functions are only partially established ${ }^{[3]}$. Selenoproteins with known functions can be generally divided into the following groups: 区antioxidative selenoproteins including five glutathione peroxidases (GPX1-4, 6), three thioredoxin reductases (TXNRD1-3), and one methionine sulfoxide reductases (MSRB1); 『selenoproteins regulating 
thyroid hormone metabolism including three iodothyronine deiodinases (DI01-3); 『Calcium-regulating selenoproteins including selenoprotein W (SELENOW) and SELENOK; 『selenoproteins regulating protein folding and endoplasmic reticulum (ER) stress including SELENOF, SELENOM, SELENON and SELENOS; \selenoproteins involving in selenium transport (SELENOP) and selenoprotein synthesis (Selenophosphate Synthetase 2, SEPHS2) ${ }^{[4]}$. Functions of SELENOT, SELENOH, SELENOI, SELENOV and SELENOO are still unclear.

While involved in various biological reactions, a notable feature of selenoproteins is their participation in cellular oxidoreductase reactions. Since oxidative stress may cause mutagenesis and genomic variability in cells, selenoproteins have long been thought to protect against cancer progression by its antioxidant role ${ }^{[5]}$. Indeed, interfering with selenoprotein biosynthesis in mice by altering Sec tRNA coding gene, Trsp, could accelerate tumor development and progression in prostate cancer ${ }^{[6]}$, colon cancer ${ }^{[7]}$ and breast cancer ${ }^{[8]}$. However, large clinical trials have generated controversial results concerning selenium supplementation for cancer prevention ${ }^{[9-11]}$. This inconsistent result could be explained by the organspecific and context-specific role of selenoproteins contributed by a hierarchical regulation of selenoprotein biosynthesis ${ }^{[12]}$. However, the potential mechanisms of individual selenoproteins in tissuespecific cancer development and progression remains obscure.

To determine the role of individual selenoproteins on pathogenesis of thyroid cancer, we carried out a multiomic data mining study and identified DIO1, GPX3, SELENOP, SELENOM, SELENOS, SELENOO and SELENOV were significantly downregulated and with a poor prognosis in thyroid cancers. Gene Ontology (GO) and Kyoto Encyclopedia of Genes and Genomes (KEGG) Enrichment were further performed for genes that are correlated with these selenoproteins to explore the potential mechanisms of their oncosuppressing role in thyroid cancers. This study provides useful information for revealing the currently unknown functions of selenoproteins and their roles in thyroid carcinogenesis.

\section{Methods}

\section{Oncomine Analysis}

Data regarding mRNA expression of 25 selenoproteins in thyroid cancers was retrieved from Oncomine (https://www.oncomine.org/resource/login.html) ${ }^{[13]}$. Primary filters for "thyroid gland carcinoma" and "cancer vs. normal analysis" were used. 12 analyses with 1147 specimens including 574 normal thyroid tissues and 573 thyroid cancer tissues were available. The database was queried for each selenoproteins individually with the filter settings as $p$-value $<0.01$, fold change $>2$, and gene ranking in the top $10 \%$.

\section{GEPIA Dataset}

GEPIA dataset (http://gepia.cancer-pku.cn/) provides access to a large collection of data from The Cancer Genome Atlas (TCGA) and the Genotype-Tissue Expression (GTEx) projects ${ }^{[14]}$. In this study, differential analysis of selenoprotein mRNA expression with a cut-off p-value of 0.01 in 512 thyroid 
cancer tissues and 337 normal thyroid tissues was validated. The relationships between expression of each selenoproteins with pathological stages of thyroid cancers were analyzed and a cut-off $p$-value of 0.05 was used.

\section{The Kaplan-Meier Plotter Analysis}

To analyze the impact of selenoproteins on survival, 502 samples from patients with thyroid cancers who underwent an average follow-up period of 240 months were retrieved from the Kaplan-Meier plotter (http://kmplot.com). They were divided into two groups according to median levels of selenoprotein expression. Kaplan-Meier survival plots were constructed to compare the two patient groups and calculate the hazard ratio (HR) with 95\% confidence intervals (Cls), and the log rank p-values.

\section{cBioPortal Analysis}

Genes that are correlated with each selenoproteins were retrieved in a papillary thyroid cancer (PTC) dataset (496 samples, TCGA Cell 2014) from cBioPortal database (https://www.cbioportal.org/) using the co-expression tool ${ }^{[15]}$. These genes harbor a correlation with Spearman's higher than 0.4 or lower than 0.4 .

Correlation between different selenoproteins in thyroid cancers were also analyzed using the cocorrelation tool on cBioportal database.

\section{Gene Ontology and Pathway Enrichment Analysis}

Functions and pathways enrichment of genes that are correlated with each selenoproteins were analyzed using DAVID (https://david.ncifcrf.gov/) ${ }^{[16]}$ and KEGG (https://genome.jp/kegg/) ${ }^{[17]}$ online databases with $p$-value $<0.05$ as the cut-off criterion.

\section{Results}

\section{Selenoprotein mRNA Expression Analysis in Thyroid Cancers}

To explore the expression pattern of selenoproteins in thyroid cancers, a meta-analysis of transcriptomic profiling data using Oncomine database was performed (Fig. 1). We observed DIO2, GPX3, SELENOP, SELENOM, SELENOS and SELENOO were underexpressed and GPX1 was overexpressed in thyroid cancer tissues. Since samples retrieved from distinct databases are different, we used another online platform, GEPIA, to confirm the results above. As shown in Fig. 2, a significantly low expression of DIO1, DIO3, GPX3, SELENOO, and SELENOV and a considerably high expression of GPX1 were observed in thyroid cancer tissues. The expressions of DIO2, SELENOP, SELENOM, and SELENOS were also reduced in thyroid cancer tissues, although no statistical significance was observed. 


\section{The Prognostic Analysis of Selenoproteins in Thyroid Cancers}

To investigate the extent to which selenoprotein expression is associated with prognosis, we detected the expression of each selenoprotein individually in different stages of thyroid cancer using GEPIA platform. As shown in Fig. 3, a negative correlation with pathological stages was observed in DIO1, DIO2, DIO3, GPX3, TXNRD2, SELENOK, SELENOP, SELENOS, SELENOV, SEPHS2 and MSRB1, while a positive correlation was seen in GPX4.

Since the overall 5-year survival rate of thyroid cancer patients is generally well with $98 \%{ }^{[18]}$, we thus compare the recurrence-free survival (RFS) of patients with high and low expression of each selenoprotein by Kaplan-Meier plotter analysis. As shown in Table 1 and Figure S1, low expression of DI01, GPX2, GPX3, TXNRD2, SELENOF, SELENOK, SELENOM, SELENOO, SELENOP, SELENOS, SELENOV, SELENOW and, and high expression of SELENOI and SEPHS2 were associated with worse RFS. 
Table 1

Association of selenoproteins expression and survival of thyroid cancer patients (Kaplan-Meier Plotter).

\begin{tabular}{|llll|}
\hline Name & Endpoint & P-value & HR [95\% Clow. Cl $\left.{ }^{\text {UP }}\right]$ \\
\hline DIO1 & Recurrence-free survival & 0.0085 & $0.35[0.16-0.79]$ \\
\hline DIO2 & Recurrence-free survival & 0.089 & $0.41[0.14-1.19]$ \\
\hline DIO3 & Recurrence-free survival & 0.063 & $0.49[0.22-1.06]$ \\
\hline GPX1 & Recurrence-free survival & 0.075 & $0.50[0.23-1.09]$ \\
\hline GPX2 & Recurrence-free survival & 0.0037 & $0.3[0.13-0.71]$ \\
\hline GPX3 & Recurrence-free survival & 0.0046 & $0.35[0.16-0.75]$ \\
\hline GPX4 & Recurrence-free survival & 0.15 & $0.55[0.24-1.25]$ \\
\hline GPX6 & Recurrence-free survival & 0.21 & $1.67[0.74-3.74]$ \\
\hline TXNRD1 & Recurrence-free survival & 0.3 & $1.66[0.63-4.40]$ \\
\hline TXNRD2 & Recurrence-free survival & 0.00066 & $0.23[0.09-0.58]$ \\
\hline TXNRD3 & Recurrence-free survival & 0.057 & $0.48[0.22-1.04]$ \\
\hline SELENOF & Recurrence-free survival & 0.0015 & $0[0-$ Inf] \\
\hline SELENOH & Recurrence-free survival & 0.12 & $0.54[0.25-1.18]$ \\
\hline SELENOI & Recurrence-free survival & 0.0096 & $2.86[1.24-6.58]$ \\
\hline SELENOK & Recurrence-free survival & 0.00022 & $0.25[0.11-0.56]$ \\
\hline SELENOM & Recurrence-free survival & 0.015 & $0.36[0.15-0.85]$ \\
\hline SELENON & Recurrence-free survival & 0.14 & $0.56[0.26-1.21]$ \\
\hline SELENOO & Recurrence-free survival & 0.014 & $0.39[0.18-0.85]$ \\
\hline SELENOP & Recurrence-free survival & 0.034 & $0.29[0.09-0.98]$ \\
\hline SELENOS & Recurrence-free survival & 0.041 & $0.46[0.21-0.99]$ \\
\hline SELENOT & Recurrence-free survival & 0.12 & $0.44[0.15-1.29]$ \\
\hline SELENOV & Recurrence-free survival & 0.0044 & $0.29[0.12-0.72]$ \\
\hline SELENOW & Recurrence-free survival & 0.0074 & $0.32[0.14-0.77]$ \\
\hline SEPHS2 & Recurrence-free survival & 0.032 & $2.62[1.05-6.54]$ \\
\hline MSRB1 & Recurrence-free survival & 0.25 & $1.59[0.72-3.50]$ \\
\hline
\end{tabular}


In order to identify selenoproteins that are clinically significant in thyroid cancers, we integrated the above selenoprotein expression data and prognostic data and found DI01, GPX3, SELENOP, SELENOM SELENOS, SELENOO and SELENOV were downregulated and with a poor prognosis in thyroid cancers. These results indicated an anti-tumor role of these selenoproteins in thyroid cancers, and we thus performed further studies to predict their biological functions and related pathways.

\section{Gene Ontology and Pathway Enrichment of Genes Correlated with DI01, GPX3, SELENOP, SELENOM SELENOS, SELENOO and SELENOV in Thyroid Cancers}

To identify genes that are correlated with DI01, GPX3, SELENOP, SELENOM SELENOS, SELENOO and SELENOV, we performed co-expression analysis (RNA Seq V2 RSEM) using cBioportal database. The correlated genes of DI01, GPX3, SELENOP, SELENOM, SELENOS, SELENOO and SELENOV with a Spearman's correlation higher than 0.4 or lower than -0.4 were listed in Table S1-S7, respectively. The top 200 genes on the correlation ranks were selected and GO analysis were performed using DAVID. As for DI01, 35 biological processes including GO: 0045926 (negative regulation of growth) were enriched in the top 200 DI01-positive-correlated genes and 23 biological processes including G0:0001525 (angiogenesis) and GO:0007155 (cell adhesion) were enriched in the top 200 DIO1-negatively-correlated genes by $\mathrm{GO}$ analysis (Table S8). The top 5 processes on each list were shown in Fig. 4A. In addition, KEGG analysis identified 7 pathways related to DI01 in thyroid cancers, including hsa04978: Mineral absorption, hsa04918: Thyroid hormone synthesis, hsa04922: Glucagon signaling pathway, hsa04977: Vitamin digestion and absorption, hsa04020: Calcium signaling pathway, hsa00980: Metabolism of xenobiotics by cytochrome P450, and hsa05205: Proteoglycans in cancer (Table 2). 
Table 2

KEGG analysis of genes correlated with DIO1, GPX3, SELENOP, SELENOM SELENOS, SELENOO and SELENOV

\begin{tabular}{|c|c|c|c|c|}
\hline & Term & Count & PValue & $\begin{array}{l}\text { Fold } \\
\text { Enrichment }\end{array}$ \\
\hline \multirow{7}{*}{ DI01 } & Mineral absorption & 5 & 0.001 & 10.56 \\
\hline & Thyroid hormone synthesis & 5 & 0.006 & 6.64 \\
\hline & Glucagon signaling pathway & 5 & 0.021 & 4.69 \\
\hline & Vitamin digestion and absorption & 3 & 0.022 & 12.68 \\
\hline & Calcium signaling pathway & 6 & 0.041 & 3.11 \\
\hline & Metabolism of xenobiotics by cytochrome P450 & 6 & 0.002 & 6.49 \\
\hline & Proteoglycans in cancer & 8 & 0.011 & 3.20 \\
\hline \multirow[t]{6}{*}{ GPX3 } & Mineral absorption & 5 & 0.001 & 10.15 \\
\hline & Valine, leucine and isoleucine biosynthesis & 2 & 0.043 & 44.67 \\
\hline & Axon guidance & 9 & 0.000 & 5.80 \\
\hline & Ras signaling pathway & 9 & 0.005 & 3.26 \\
\hline & Adherens junction & 5 & 0.010 & 5.77 \\
\hline & Rap1 signaling pathway & 8 & 0.013 & 3.12 \\
\hline \multirow[t]{13}{*}{ SELM } & Parkinson's disease & 14 & 0.000 & 11.50 \\
\hline & Oxidative phosphorylation & 13 & 0.000 & 11.40 \\
\hline & Alzheimer's disease & 14 & 0.000 & 9.72 \\
\hline & Non-alcoholic fatty liver disease (NAFLD) & 13 & 0.000 & 10.04 \\
\hline & Huntington's disease & 13 & 0.000 & 7.89 \\
\hline & Metabolic pathways & 26 & 0.000 & 2.49 \\
\hline & Cardiac muscle contraction & 5 & 0.003 & 7.77 \\
\hline & Ubiquitin mediated proteolysis & 9 & 0.000 & 6.85 \\
\hline & ErbB signaling pathway & 6 & 0.001 & 7.18 \\
\hline & Focal adhesion & 8 & 0.003 & 4.05 \\
\hline & Epithelial cell signaling in Helicobacter pylori infection & 5 & 0.003 & 7.78 \\
\hline & Small cell lung cancer & 5 & 0.008 & 6.13 \\
\hline & TNF signaling pathway & 5 & 0.018 & 4.87 \\
\hline
\end{tabular}




\begin{tabular}{|c|c|c|c|c|}
\hline & Term & Count & PValue & $\begin{array}{l}\text { Fold } \\
\text { Enrichment }\end{array}$ \\
\hline & Pathways in cancer & 9 & 0.030 & 2.39 \\
\hline & Proteoglycans in cancer & 6 & 0.039 & 3.13 \\
\hline & Salmonella infection & 4 & 0.043 & 5.02 \\
\hline & TGF-beta signaling pathway & 4 & 0.044 & 4.96 \\
\hline & Regulation of actin cytoskeleton & 6 & 0.047 & 2.98 \\
\hline SELS & Vibrio cholerae infection & 4 & 0.026 & 6.08 \\
\hline & Ribosome & 6 & 0.027 & 3.49 \\
\hline & Protein export & 7 & 0.000 & 24.06 \\
\hline & Spliceosome & 8 & 0.001 & 4.76 \\
\hline & Proteasome & 9 & 0.000 & 16.17 \\
\hline & Non-alcoholic fatty liver disease (NAFLD) & 12 & 0.000 & 6.28 \\
\hline & Parkinson's disease & 13 & 0.000 & 7.24 \\
\hline & Alzheimer's disease & 13 & 0.000 & 6.12 \\
\hline & Huntington's disease & 13 & 0.000 & 5.35 \\
\hline & Oxidative phosphorylation & 14 & 0.000 & 8.32 \\
\hline & Lysine degradation & 4 & 0.009 & 8.82 \\
\hline & Renal cell carcinoma & 4 & 0.018 & 6.95 \\
\hline & Salmonella infection & 4 & 0.033 & 5.53 \\
\hline & Prostate cancer & 4 & 0.039 & 5.21 \\
\hline & Leukocyte transendothelial migration & 4 & 0.075 & 3.99 \\
\hline & Notch signaling pathway & 5 & 0.000 & 11.94 \\
\hline & HIF-1 signaling pathway & 5 & 0.009 & 5.97 \\
\hline & Platelet activation & 5 & 0.024 & 4.41 \\
\hline & Ubiquitin mediated proteolysis & 5 & 0.029 & 4.18 \\
\hline & cAMP signaling pathway & 5 & 0.089 & 2.90 \\
\hline & Adherens junction & 6 & 0.000 & 9.69 \\
\hline & Focal adhesion & 6 & 0.031 & 3.34 \\
\hline
\end{tabular}




\begin{tabular}{|c|c|c|c|c|}
\hline & Term & Count & PValue & $\begin{array}{l}\text { Fold } \\
\text { Enrichment }\end{array}$ \\
\hline & Regulation of actin cytoskeleton & 6 & 0.033 & 3.28 \\
\hline & HTLV-I infection & 6 & 0.065 & 2.71 \\
\hline & Thyroid hormone signaling pathway & 7 & 0.000 & 6.98 \\
\hline & Pathways in cancer & 10 & 0.005 & 2.92 \\
\hline \multirow[t]{8}{*}{ SELO } & Pyrimidine metabolism & 4 & 0.040 & 5.14 \\
\hline & Purine metabolism & 5 & 0.043 & 3.69 \\
\hline & RNA transport & 8 & 0.002 & 4.21 \\
\hline & TNF signaling pathway & 6 & 0.006 & 5.08 \\
\hline & $\begin{array}{l}\text { Signaling pathways regulating pluripotency of stem } \\
\text { cells }\end{array}$ & 6 & 0.017 & 3.88 \\
\hline & Protein processing in endoplasmic reticulum & 6 & 0.036 & 3.21 \\
\hline & Cell cycle & 5 & 0.045 & 3.65 \\
\hline & Endocytosis & 7 & 0.046 & 2.63 \\
\hline \multirow[t]{8}{*}{ SELV } & Insulin resistance & 5 & 0.027 & 4.30 \\
\hline & Adipocytokine signaling pathway & 4 & 0.037 & 5.31 \\
\hline & Adherens junction & 6 & 0.001 & 7.09 \\
\hline & Proteoglycans in cancer & 9 & 0.002 & 3.78 \\
\hline & ECM-receptor interaction & 6 & 0.003 & 5.79 \\
\hline & Pathways in cancer & 11 & 0.016 & 2.35 \\
\hline & Hippo signaling pathway & 6 & 0.032 & 3.33 \\
\hline & $\begin{array}{l}\text { Arrhythmogenic right ventricular cardiomyopathy } \\
\text { (ARVC) }\end{array}$ & 4 & 0.043 & 5.01 \\
\hline
\end{tabular}

In terms of GPX3, GO analysis revealed that 41 biological processes including G0: 0045926 (negative regulation of growth) were enriched in the top 200 GPX3-positive-correlated genes and 23 biological processes including G0:0007165 (signal transduction) and GO: 0000165 (MAPK cascade) were enriched in the top 200 GPX3-negative-correlated genes (Table S9). The top 5 processes on each list were shown in Fig. 4B. KEGG analysis enriched these genes in 6 pathways, including hsa04978: Mineral absorption, hsa00290: Valine, leucine and isoleucine biosynthesis, hsa04360: Axon guidance, hsa04014: Ras signaling pathway, hsa04520: Adherens junction, and hsa04015: Rap1 signaling pathway (Table 2). 
With regards to SELENOP, GO and KEGG enrichment failed to identify any functions and pathways except for the biological process GO: 0018105 (peptidyl-serine phosphorylation), which is involved in selenoprotein biosynthesis (data not shown).

Concerning SELENOM, GO analysis identified 15 biological processes enriched in the top 200 SELENOMpositive-correlated genes (Table S10). These processes are mainly involved in mitochondrial function. 46 biological processes were enriched in the top 200 SELENOM-negative-correlated genes (Table S10). These processes are mainly involved in gene transcription and protein ubiquitination, while GO: 0007249 (I-kappaB kinase/NF-kappaB signaling), GO: 0051301 (cell division), GO: 0007219 (Notch signaling pathway), and GO: 0016477 (cell migration) also act in tumorigenesis. The top 5 processes on each list were shown in Fig. 4C. KEGG analysis identified 18 pathways related to SELENOM in thyroid cancers (Table 2). Among these pathways, hsa04012: ErbB signaling pathway, hsa04510: Focal adhesion, hsa04668: TNF signaling pathway, hsa05200: Pathways in cancer, hsa05205: Proteoglycans in cancer, and hsa04350: TGF-beta signaling pathway play a major role in tumor progression.

Regarding SELENOS, GO analysis identified 46 biological processes enriched in the top 200 SELENOSpositive-correlated genes and 51 biological processes enriched in the top 200 SELENOS-negativecorrelated genes (Table S11). The top 5 processes on each list were shown in Fig. 4D. These processes are mainly involved in mitochondrial translation, protein ubiquitination, gene transcription and viral process. KEGG analysis identified 25 pathways related to SELENOS in thyroid cancers. Among them hsa04520: Adherens junction, hsa04330: Notch signaling pathway, hsa05200: Pathways in cancer, hsa05211: Renal cell carcinoma, hsa04510: Focal adhesion, and hsa05215: Prostate cancer have been implicated in cancer pathogenesis (Table 2).

In terms of SELENOO, 7 biological processes enriched in the top 200 SELENOO-positive-correlated genes and 41 biological processes enriched in the top 200 SELENOO-negative-correlated genes (Table S12) were identified by GO analysis. The top 5 biological processes on each list were shown in Fig. 4E. These processes are mainly involved in mitochondrial translation, endoplasmic reticulum stress, protein catabolism, and telomere maintenance. KEGG analysis identified 8 pathways related to SELENOO in thyroid cancers, including hsa03013: RNA transport, hsa04668: TNF signaling pathway, hsa04550: Signaling pathways regulating pluripotency of stem cells, hsa04141: Protein processing in endoplasmic reticulum, hsa04110: Cell cycle, hsa04144: Endocytosis, hsa00240: Pyrimidine metabolism, and hsa00230: Purine metabolism (Table 2).

With regards to SELENOV, 22 biological processes including G0:0055085 (transmembrane transport) and GO:0055114 (oxidation-reduction process) were enriched in the top 200 SELENOV-positive-correlated genes and 35 biological processes including GO: 0098609 (cell-cell adhesion), G0:0042060 (wound healing), GO: 0007155 (cell adhesion) and GO: 2001237 (negative regulation of extrinsic apoptotic signaling pathway) were enriched in the top 200 SELENOV-negatively-correlated genes by GO analysis (Table S13). The top 5 processes on each list were shown in Fig. 4F. In addition, 8 pathways including hsa04931: Insulin resistance, hsa04920: Adipocytokine signaling pathway, hsa04520: Adherens junction, 
hsa05205: Proteoglycans in cancer, hsa04512: ECM-receptor interaction, hsa05200: Pathways in cancer, hsa04390: Hippo signaling pathway, and hsa05412: Arrhythmogenic right ventricular cardiomyopathy (ARVC) were identified to be correlated with SELENOV (Table 2).

\section{Correlations of Selenoproteins with Each Other}

Next, we calculated the Spearman's rank correlations of selenoproteins with each other by analyzing their mRNA expression (RNA Seq V2 RSEM) using cBioPortal online database for thyroid cancers. The results indicated a significant and positive correlations $(r \geq 0.6)$ in the following selenoproteins: DIO1 with SELENOV; GPX1 with GPX4, SELENOW, and SELENOH; TXNRD2 with SELENOO; SELENOK with SELENOS (Fig. 4G).

\section{Discussion}

Low serum and tissue levels of selenium are associated with increased incidence and advanced stage of thyroid cancers ${ }^{[2]}$. In response to changes in selenium supply, a hierarchy of selenoprotein biosynthesis allows tissue-specific fine-tuning of the 25 selenoproteins ${ }^{[12]}$. However, the role of individual selenoproteins on thyroid tumorigenesis and metastasis remains unclear. Here, we found an underexpression of DI01, GPX3, SELENOP, SELENOM SELENOS, SELENOO and SELENOV in thyroid cancers and their downregulation was associated with a poor prognosis. The connection of a decreased expression to poor tumor prognosis strongly indicates a potential onco-suppressing role of these selenoproteins in thyroid cancers.

DIOs play an important role in the regulation of thyroid hormone metabolism and intracellular action. DIO2 convert thyroxine (T4) to the active hormone, triiodothyronine (T3) through outer ring deiodination; while DIO3 inactivate THs through inner ring deiodinase. DIO1 involves in both inner and outer ring deiodinations and is viewed as a scavenger enzyme in iodine recycling from the inactive iodothyronines [19]. Since thyroid hormones regulate cellular growth, development and differentiation, it is not surprising that expression of these deiodinases altered in various cancers, such as breast cancers, renal cancers, liver cancers, lung cancers, brain tumors, and thyroid cancers ${ }^{[20-21]}$. Mechanistic studies to determine how exactly these deiodinases modify tumor growth, however, are still lacking. In the current study, a tumor suppressing role of DIO1 was established and GO analysis showed DI01-negative-correlated genes were mainly enriched in biological processes including angiogenesis and cell adhesion. The antimetastatic effect of DI01 was in fact comparable to that observed in renal cancer cells, in which ectopic expression of DIO1 inhibit proliferation and migration through regulating the expression of integrins and their ligands, including collagens, fibronectin, laminin, and vitronectin ${ }^{[22]}$. In addition, biological process GO: 0045926 (negative regulation of growth), which involves 5 members of metallothionein (MT) family, was identified to be positively related with DI01. MTs are a family of low molecular and cysteine-rich proteins regulating heavy metal homeostasis and oxidation-reduction reactions ${ }^{[23]}$. In thyroid cells, defects in MT1G and MT1M expression has been linked to malignant transformation ${ }^{[24-26]}$. Yet, the specific mechanisms how DI01 and MTs are related with each other remain unclear. Zinc plays a key role 
in thyroid hormone metabolism specially by regulating deiodinases enzyme activities ${ }^{[27]}$ and exerts anticancer role via many cellular processes such as antioxidant reactions, transcriptional regulations, DNA and RNA repair ${ }^{[28]}$. Since MTs are able to bind both Zinc and Selenium, it is possible that defects in DIO1 and MTs expression are attributed to changes of these physiology heavy metals in the context of thyroid cancers.

GPX1-4 and GPX6 are selenium-containing GPX family members that catalyze hydroperoxide reduction using GSH as reductant. Among the GPX family members, GPX3 is the only one that can be secreted into the plasma, and thus plays a critical role in reduction of extracellular ROS stress ${ }^{[29]}$. Downregulation of GPX3 by hypermethylation has been well established in thyroid carcinogenesis, while expression of other GPXs in thyroid cancer remain controversial ${ }^{[30]}$. Consistently, we found a decreased GPX3 expression connected with a poor prognosis of thyroid cancer, whereas the connection was not observed in other GPX family members. In thyroid, GPX3 is detected in the follicular lumen to protect the thyroid follicular cells from possible damage by excess $\mathrm{H} 2 \mathrm{O} 2$, an oxidative agent required for thyroid hormone synthesis [31]. GO analysis revealed signal transduction was enriched in GPX3-negative-correlated genes, which may be explained by the accumulation of hydroperoxides, particularly $\mathrm{H} 2 \mathrm{O} 2$, by GPX 3 downregulation during transformation of thyroid cancer cells ${ }^{[32]}$. In addition, MAPK signaling, a major and highly prevalent oncogenic pathway in thyroid cancers, was identified to be negatively correlated with GPX3 ${ }^{[33]}$. GPX3 has been reported to inhibit cell growth and metastasis in prostate cancer through downregulation of c-Met, which is a tyrosine kinase receptor initiating MAPK activation ${ }^{[34-35]}$. Thus, suppression of MAPK cascade by GPX3 might be mediated by c-Met, in which further studies are needed to clarify the precise mechanism. Moreover, the positive correlation of MTs and GPX3 might be explained by their responses to changes in selenium levels as discussed above.

SELENOM and SELENOS are ER-resident selenoproteins regulating protein folding and degradation, consistent with the results of our $\mathrm{GO}$ analysis ${ }^{[36]}$. In cancer cells, enhanced protein synthesis triggers ER stress and the sustained ER stress promotes tumorigenesis and metastasis ${ }^{[37]}$. Accordingly, the antitumor effects of SELENOM and SELENOS may be attributed to their protection against ER stress in thyroid cells. In addition, GO analysis revealed that SELENOM and SELENOS regulate mitochondrial translation responsible for the maintenance of energetic balance. Currently, there is no evidence implicating their expression in mitochondria. Since a direct interaction of ER and mitochondria has been reported ${ }^{[38]}$, it is possible that SELENOM and SELENOS are somehow involved in the interconnectedness of the ER and mitochondria.

To date, functions of SELENOO and SELENOV are largely unknown. Our data showed SELENOO is involved in ER stress, mitochondria translation and telomere maintenance, similar as SELENOM and SELENOS. The tumor suppressing role of SELENOO, thus, probably connects with its potential role in regulation of ER functions. SELENOV was recently reported to protect against reactive oxygen and nitrogen species-mediated ER stress and oxidative injury ${ }^{[39]}$, which is likely one of the mechanisms for its anti-cancer role. Consistently, our data showed SELENOV is related with biological processes including 
oxidation-reduction process and ER calcium ion homeostasis. Moreover, cell adhesion and angiogenesis were also shown to be negatively regulated by SELENOV, providing an anti-metastatic effect similar as DI01. In accordance with our correlation analysis, functions of SELENOV and DIO1 might be significantly correlated with each other. However, the precise mechanisms and implications in cancer progression still remain to be clarified.

Whereas well known for its role in selenium transport, SELENOP has antioxidant capacity exerting its "double-edged sword" effects in the process of cell transformation ${ }^{[40]}$. After produced in the liver, SELENOP is secreted into plasma and travel to targeted organs participating in synthesis of other selenoproteins ${ }^{[41]}$. Here, we showed SELENOP was downregulated in thyroid cancer tissues and GO analysis failed to identify any other biological processes except for selenoprotein biosynthesis. Thus, a low expression of SELENOP in thyroid cancer tissues mainly indicates a reduced local supply of selenium. However, selenium supplementation for thyroid cancer prevention might not be effective and even could be harmful, since expressions of selenoproteins are not all reduced and some even increased in the context of thyroid cancer. Thus, targeted regulation of individual selenoproteins, such as DI01, GPX3, SELENOP, SELENOM SELENOS, SELENOO and SELENOV, may serve as a potential therapeutic option for thyroid cancer.

\section{Conclusions}

A multiomic data mining study was performed to determine the role of individual selenoproteins on thyroid carcinogenesis. DI01, GPX3, SELENOP, SELENOM SELENOS, SELENOO and SELENOV were found to be significantly downregulated and associated with a poor prognosis, indicating an anti-tumor role in thyroid cancer. Our results provide useful information for uncovering the currently unknown functions of selenoproteins. With the understanding of distinct roles of selenoproteins in thyroid cancer pathogenesis, we now open up the possibility for targeted regulation of individual selenoproteins for the treatment of thyroid cancer.

\section{Abbreviations}

Glutathione Peroxidases (GPX)

Thioredoxin Reductases (TXNRD)

Methionine Sulfoxide Reductases (MSRB1)

lodothyronine Deiodinases (DIO)

Selenoprotein (SELENO)

Endoplasmic Reticulum (ER)

Selenophosphate Synthetase 2 (SEPHS2) 
Gene Ontology (GO)

Kyoto Encyclopedia of Genes and Genomes (KEGG)

\section{Declarations}

\section{Ethics approval and consent to participate}

Not applicable

\section{Consent for publication}

Not applicable

\section{Availability of data and materials}

The datasets analysed during the current study are available in the Oncomine (https://www.oncomine.org/resource/login.html), GEPIA (http://gepia.cancer-pku.cn/), Kaplan-Meier plotter (http://kmplot.com), and cBioPortal database (https://www.cbioportal.org/).

\section{Competing of Interests}

The authors declare that there is no conflict of interest regarding the publication of this paper.

\section{Funding}

The research was supported by grants from National Natural Science Foundation of China (grant No. 81500597 to JF and grant No. 82074045 to XYD), Natural Science Basic Research Plan in Shaanxi Province of China (program number 2017JM8051 to JF) and the Fundamental Research Funds for the Central Universities (fund number sxjh012019062 to JF).

\section{Author's contributions}

ZY and CP analyzed and interpreted the data regarding the expression of selenoproteins. DXY did prognosis analysis of selenoproteins in thyroid cancer. $Z Y$ retrieved the genes that are correlated with the selenoproteins. LHJ and WY performed GO and KEGG analysis for selenoprotein-related genes. FJ and LS was a major contributor in writing the manuscript. All authors read and approved the final manuscript. 


\section{Acknowledgements}

Not applicable

\section{References}

1. Haugen BR, Alexander EK, Bible KC, Doherty GM, Mandel SJ, Nikiforov YE, Pacini F, Randolph GW, Sawka AM, Schlumberger M, et al. 2015 American Thyroid Association Management Guidelines for Adult Patients with Thyroid Nodules and Differentiated Thyroid Cancer: The American Thyroid Association Guidelines Task Force on Thyroid Nodules and Differentiated Thyroid Cancer. Thyroid. 2016;26:1-133.

2. Barrea L, Gallo M, Ruggeri RM, Giacinto PD, Sesti F, Prinzi N, Adinolfi V, Barucca V, Renzelli V, Muscogiuri G, et al. Nutritional status and follicular-derived thyroid cancer: An update. Crit Rev Food Sci Nutr. 2021;61:25-59.

3. Kryukov GV, Castellano S, Novoselov SV, Lobanov AV, Zehtab O, Guigo R, Gladyshev VN. Characterization of mammalian selenoproteomes. Science. 2003;300:1439-43.

4. Papp LV, Holmgren A, Khanna KK. Selenium and selenoproteins in health and disease. Antioxid Redox Signal. 2010;12:793-5.

5. Short SP, Williams CS. Selenoproteins in Tumorigenesis and Cancer Progression. Adv Cancer Res. 2017;136:49-83.

6. Diwadkar-Navsariwala V, Prins GS, Swanson SM, Birch LA, Ray VH, Hedayat S, Lantvit DL, Diamond AM. Selenoprotein deficiency accelerates prostate carcinogenesis in a transgenic model. Proc Natl Acad Sci U S A. 2006;103:8179-84.

7. Irons R, Carlson BA, Hatfield DL, Davis CD. Both selenoproteins and low molecular weight selenocompounds reduce colon cancer risk in mice with genetically impaired selenoprotein expression. J Nutr. 2006;136:1311-7.

8. Hudson TS, Carlson BA, Hoeneroff MJ, Young HA, Sordillo L, Muller WJ, Hatfield DL, Green JE. Selenoproteins reduce susceptibility to DMBA-induced mammary carcinogenesis. Carcinogenesis. 2012;33:1225-30.

9. Duffield-Lillico AJ, Dalkin BL, Reid ME, Turnbull BW, Slate EH, Jacobs ET, Marshall JR, Clark LC. Nutritional Prevention of Cancer Study G: Selenium supplementation, baseline plasma selenium status and incidence of prostate cancer: an analysis of the complete treatment period of the Nutritional Prevention of Cancer Trial. BJU Int. 2003;91:608-12.

10. Klein EA, Thompson IM Jr, Tangen CM, Crowley JJ, Lucia MS, Goodman PJ, Minasian LM, Ford LG, Parnes HL, Gaziano JM, et al. Vitamin E and the risk of prostate cancer: the Selenium and Vitamin E Cancer Prevention Trial (SELECT). JAMA. 2011;306:1549-56.

11. Lance P, Alberts DS, Thompson PA, Fales L, Wang F, San Jose J, Jacobs ET, Goodman PJ, Darke AK, Yee M, et al. Colorectal Adenomas in Participants of the SELECT Randomized Trial of Selenium and 
Vitamin E for Prostate Cancer Prevention. Cancer Prev Res (Phila). 2017;10:45-54.

12. Schomburg L, Schweizer U. Hierarchical regulation of selenoprotein expression and sex-specific effects of selenium. Biochim Biophys Acta. 2009;1790:1453-62.

13. Rhodes DR, Yu J, Shanker K, Deshpande N, Varambally R, Ghosh D, Barrette T, Pandey A, Chinnaiyan AM. ONCOMINE: a cancer microarray database and integrated data-mining platform. Neoplasia. 2004;6:1-6.

14. Tang Z, Li C, Kang B, Gao G, Li C, Zhang Z. GEPIA: a web server for cancer and normal gene expression profiling and interactive analyses. Nucleic Acids Res. 2017;45:W98-102.

15. Cerami E, Gao J, Dogrusoz U, Gross BE, Sumer SO, Aksoy BA, Jacobsen A, Byrne CJ, Heuer ML, Larsson $\mathrm{E}$, et al. The cBio cancer genomics portal: an open platform for exploring multidimensional cancer genomics data. Cancer Discov. 2012;2:401-4.

16. Dennis G Jr, Sherman BT, Hosack DA, Yang J, Gao W, Lane HC, Lempicki RA. DAVID: Database for Annotation, Visualization, and Integrated Discovery. Genome Biol. 2003;4:P3.

17. Kanehisa M, Sato $Y$, Kawashima M, Furumichi $M$, Tanabe M. KEGG as a reference resource for gene and protein annotation. Nucleic Acids Res. 2016;44:D457-62.

18. Haugen BR. 2015 American Thyroid Association Management Guidelines for Adult Patients with Thyroid Nodules and Differentiated Thyroid Cancer: What is new and what has changed? Cancer. 2017;123:372-81.

19. Bianco AC, Salvatore D, Gereben B, Berry MJ, Larsen PR. Biochemistry, cellular and molecular biology, and physiological roles of the iodothyronine selenodeiodinases. Endocr Rev. 2002;23:38-89.

20. Goemann IM, Romitti M, Meyer ELS, Wajner SM, Maia AL. Role of thyroid hormones in the neoplastic process: an overview. Endocr Relat Cancer. 2017;24:R367-85.

21. Casula S, Bianco AC. Thyroid hormone deiodinases and cancer. Front Endocrinol (Lausanne). 2012;3:74.

22. Poplawski P, Rybicka B, Boguslawska J, Rodzik K, Visser TJ, Nauman A, Piekielko-Witkowska A. Induction of type 1 iodothyronine deiodinase expression inhibits proliferation and migration of renal cancer cells. Mol Cell Endocrinol. 2017;442:58-67.

23. Eckschlager T, Adam V, Hrabeta J, Figova K, Kizek R. Metallothioneins and cancer. Curr Protein Pept Sci. 2009;10:360-75.

24. Ferrario C, Lavagni P, Gariboldi M, Miranda C, Losa M, Cleris L, Formelli F, Pilotti S, Pierotti MA, Greco A. Metallothionein $1 \mathrm{G}$ acts as an oncosupressor in papillary thyroid carcinoma. Lab Invest. 2008;88:474-81.

25. Fu J, Lv H, Guan H, Ma X, Ji M, He N, Shi B, Hou P. Metallothionein $1 \mathrm{G}$ functions as a tumor suppressor in thyroid cancer through modulating the PI3K/Akt signaling pathway. BMC Cancer. 2013;13:462.

26. Chen Y, Quan R, Bhandari A, Chen Z, Guan Y, Xiang J, You J, Teng L. Low metallothionein 1M (MT1M) is associated with thyroid cancer cell lines progression. Am J Transl Res. 2019;11:1760-70. 
27. Severo JS, Morais JBS, de Freitas TEC, Andrade ALP, Feitosa MM, Fontenelle LC, de Oliveira ARS, Cruz KJC. do Nascimento Marreiro D: The Role of Zinc in Thyroid Hormones Metabolism. Int J Vitam Nutr Res. 2019;89:80-8.

28. Skrajnowska D, Bobrowska-Korczak B. Role of Zinc in Immune System and Anti-Cancer Defense Mechanisms. Nutrients 2019, 11.

29. Kipp AP. Selenium-Dependent Glutathione Peroxidases During Tumor Development. Adv Cancer Res. 2017;136:109-38.

30. Zhao H, Li J, Li X, Han C, Zhang Y, Zheng L, Guo M. Silencing GPX3 Expression Promotes Tumor Metastasis in Human Thyroid Cancer. Curr Protein Pept Sci. 2015;16:316-21.

31. Schmutzler C, Mentrup B, Schomburg L, Hoang-Vu C, Herzog V, Kohrle J. Selenoproteins of the thyroid gland: expression, localization and possible function of glutathione peroxidase 3 . Biol Chem. 2007;388:1053-9.

32. Liou GY, Storz P. Reactive oxygen species in cancer. Free Radic Res. 2010;44:479-96.

33. Xing M. Molecular pathogenesis and mechanisms of thyroid cancer. Nat Rev Cancer. 2013;13:18499.

34. Zhang Y, Xia M, Jin K, Wang S, Wei H, Fan C, Wu Y, Li X, Li X, Li G, et al. Function of the c-Met receptor tyrosine kinase in carcinogenesis and associated therapeutic opportunities. Mol Cancer. 2018;17:45.

35. Yu YP, Yu G, Tseng G, Cieply K, Nelson J, Defrances M, Zarnegar R, Michalopoulos G, Luo JH. Glutathione peroxidase 3, deleted or methylated in prostate cancer, suppresses prostate cancer growth and metastasis. Cancer Res. 2007;67:8043-50.

36. Papp LV, Holmgren A, Khanna KK. Selenium and selenoproteins in health and disease. Antioxid Redox Signal. 2010;12:793-5.

37. Cubillos-Ruiz JR, Bettigole SE, Glimcher LH. Tumorigenic and Immunosuppressive Effects of Endoplasmic Reticulum Stress in Cancer. Cell. 2017;168:692-706.

38. Lombardi AA, Elrod JW. Mediating ER-mitochondrial cross-talk. Science. 2017;358:591-2.

39. Zhang X, Xiong W, Chen LL, Huang JQ, Lei XG. Selenoprotein V protects against endoplasmic reticulum stress and oxidative injury induced by pro-oxidants. Free Radic Biol Med. 2020;160:670-9.

40. Perez S, Talens-Visconti R, Rius-Perez S, Finamor I, Sastre J. Redox signaling in the gastrointestinal tract. Free Radic Biol Med. 2017;104:75-103.

41. Perez S, Talens-Visconti R, Rius-Perez S, et al. Redox signaling in the gastrointestinal tract. Free Radic Biol Med. 2017;104:75-103.

\section{Figures}




\begin{tabular}{|c|c|c|c|c|c|c|c|c|c|c|c|c|c|c|}
\hline & & & \multicolumn{12}{|c|}{ Tissue type } \\
\hline Gene & Median rank & $\mathrm{P}$ & 1 & 2 & 3 & 4 & 5 & 6 & 7 & 8 & 9 & 10 & 11 & 12 \\
\hline DI01 & 3045.0 & 0.062 & & & & & & & & & & & & \\
\hline DI02 & 583.5 & 0.002 & & & & & & & & & & & & \\
\hline DI03 & 78920 & 0.364 & & & & & & & & & & & & \\
\hline GPX1 & 1889.5 & 0.030 & & & & & & & & & & & & \\
\hline GPX2 & 5615.5 & 0.190 & & & & & & & & & & & & \\
\hline GPX3 & 2534.5 & 0.040 & & & & & & & & & & & & \\
\hline GPX4 & 3644.0 & 0.092 & & & & & & & & & & & & \\
\hline GPX6 & 10026.5 & 0.477 & & & & & & & & & & & & \\
\hline TXNRD1 & 4846. 0 & 0.189 & & & & & & & & & & & & \\
\hline TXNRD2 & 69825 & 0.417 & & & & & & & & & & & & \\
\hline TXNRD3 & 5267.0 & 0.263 & & & & & & & & & & & & \\
\hline MSRB1 & 7333.5 & 0.423 & & & & & & & & & & & & \\
\hline SELENOK & 9300.0 & 0.463 & & & & & & & & & & & & \\
\hline SELENOW & 5584.0 & 0.226 & & & & & & & & & & & & \\
\hline SEPHS2 & 6798.5 & 0.351 & & & & & & & & & & & & \\
\hline SELENOP & 2550.5 & 0.026 & & & & & & & & & & & & \\
\hline SELENOF & 61920 & 0.179 & & & & & & & & & & & & \\
\hline SELENOM & 594.0 & 0.009 & & & & & & & & & & & & \\
\hline SELENON & 7528.5 & 0.339 & & & & & & & & & & & & \\
\hline SELENOS & 2654.5 & 0.047 & & & & & & & & & & & & \\
\hline SELENOH & 10238.0 & 0.492 & & & & & & & & & & & & \\
\hline SELENOI & 66620 & 0.156 & & & & & & & & & & & & \\
\hline SELENOO & 411.0 & 0.005 & & & & & & & & & & & & \\
\hline SELENOT & 6420.5 & 0.265 & & & & & & & & & & & & \\
\hline SELENOV & 4579.0 & 0.115 & & & & & & & & & & & & \\
\hline
\end{tabular}

Legend

1. Follicular Variant Thyroid Gland Papillary Carcinoma vs. Normal

Giordano Thyroid, Clin Cancer Res, 2006

2. Tall Cell Variant Thyroid Gland Papillary Carcinoma vs. Normal

Giordano Thyroid, Clin Cancer Res, 2006

3. Thyroid Gland Follicular Carcinoma vs. Normal Giordano Thyroid, Clin Cancer Res, 2006

4. Thyroid Gland Oncocytic Follicular Carcinoma vs. Normal

Giordano Thyroid, Clin Cancer Res, 2006

5. Thyroid Gland Papillary Carcinoma vs. Normal Giordano Thyroid, Clin Cancer Res, 2006

6. Thyroid Gland Undifferentiated (Anaplastic)

Carcinoma vs. Normal

Giordano Thyroid, Clin Cancer Res, 2006

7. Thyroid Gland Papillary Carcinoma vs. Normal

He Thyroid, Proc Natl Acad Sci U S A, 2005

8. Follicular Variant Thyroid Gland Papillary

Carcinoma vs. Normal

TCGA Thyroid, No Associated Paper, 2013

9. Tall Cell Variant Thyroid Gland Papillary Carcinoma

vs. Normal

TCGA Thyroid, No Associated Paper, 2013

10. Thyroid Gland Carcinoma vs. Normal

TCGA Thyroid, No Associated Paper, 2013

11. Thyroid Gland Papillary Carcinoma vs. Normal

TCGA Thyroid, No Associated Paper, 2013

12. Thyroid Gland Papillary Carcinoma vs. Normal

Vasko Thyroid, Proc Natl Acad Sci U S A, 2007

Under-expressed 15102525105

Over-expressed

\section{Figure 1}

Meta-analysis of gene expression profiling for the 25 selenoproteins in thyroid cancers using Oncomine database. With a cut-off $p$-value $<0.01$ and fold change $>2$, underexpressed selenoproteins were shown in blue and overexpressed selenoproteins were shown in red. 

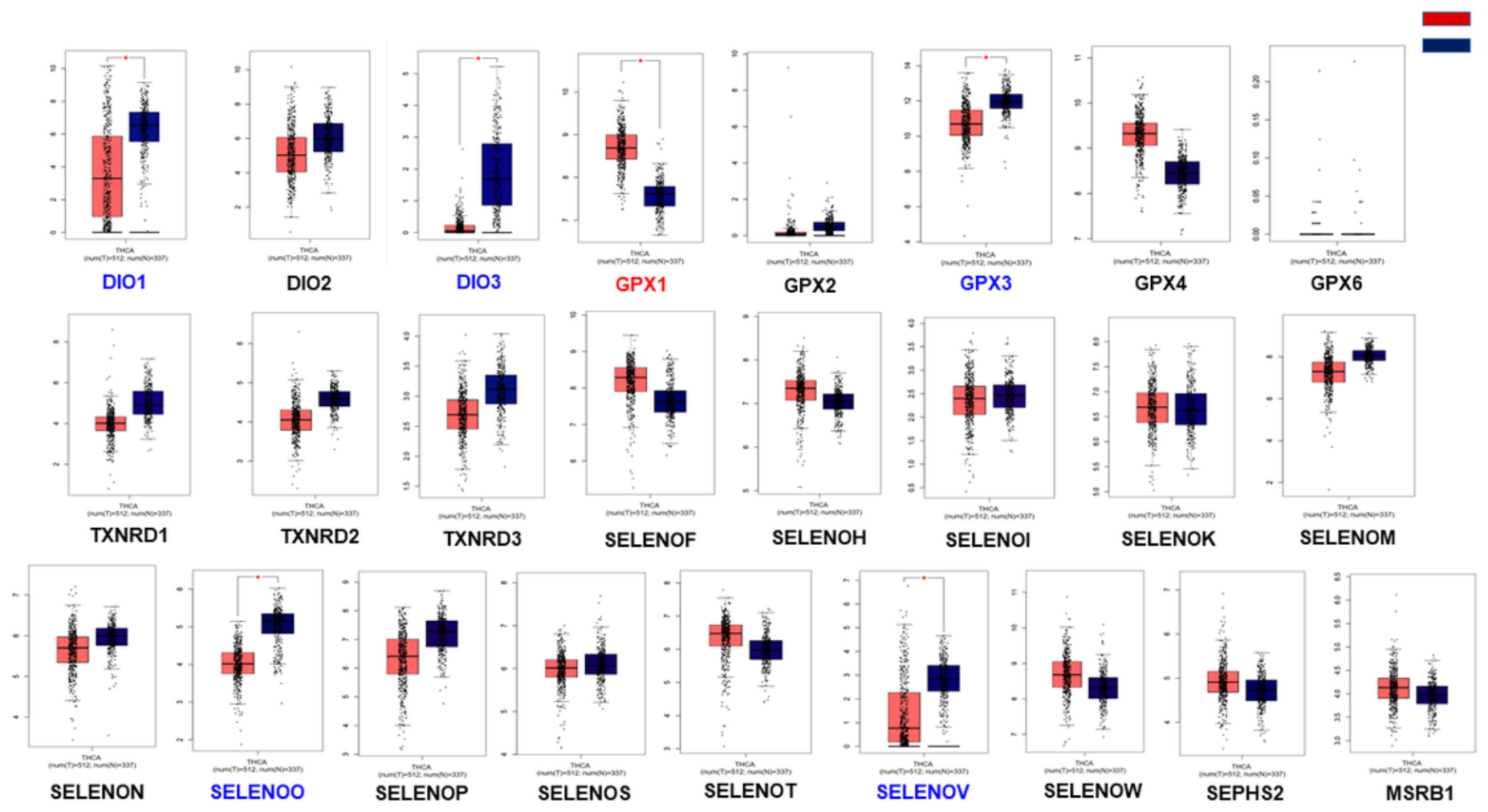

\section{Figure 2}

Selenoprotein mRNA expression analysis in thyroid cancers using GEPIA platform. With a cut-off p-value of 0.01 , underexpressed selenoproteins were shown in blue and overexpressed selenoproteins were shown in red (T: thyroid cancer tissues; $\mathrm{N}$ : non-malignant thyroid tissues; ${ }^{*} \mathrm{p} \leq 0.01$ ). 


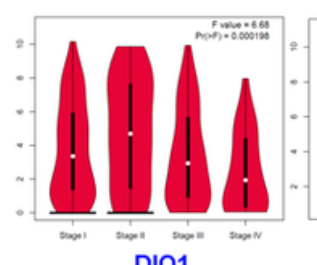

DIO1

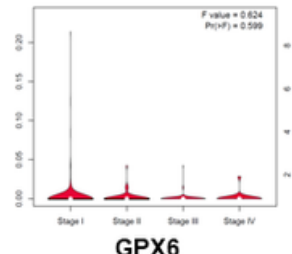

GPX6

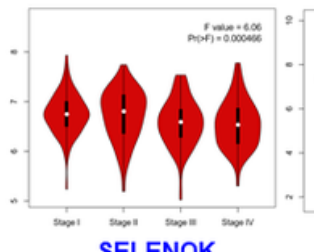

SELENOK
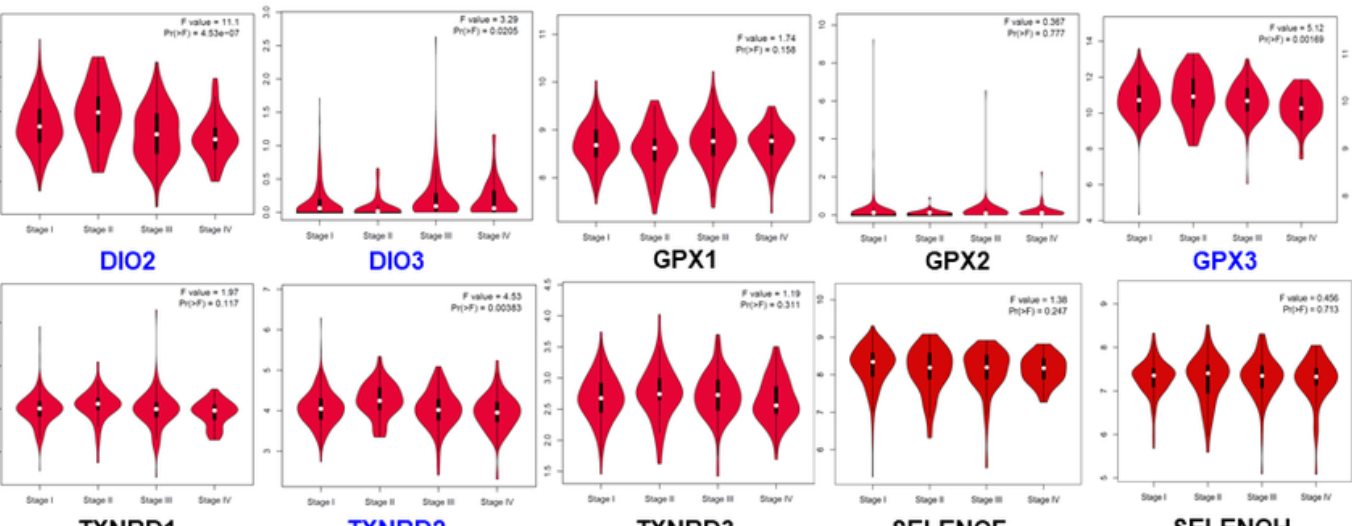

SELENOF

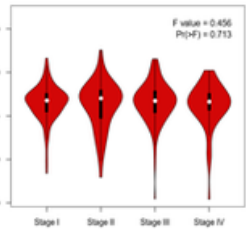

SELENOH

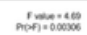
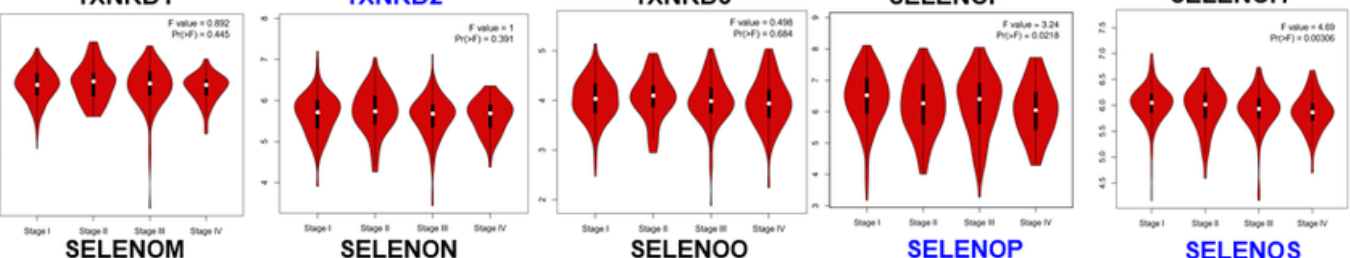

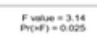

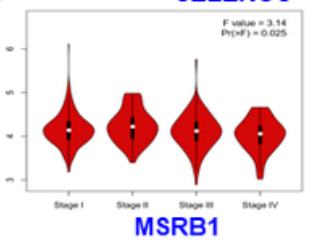

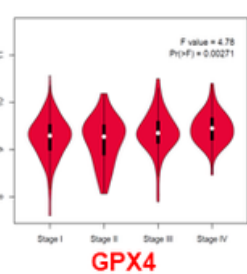

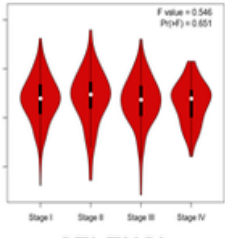

SELENOI

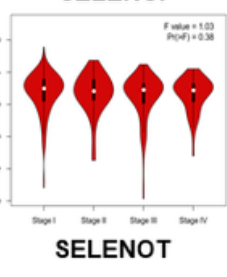

Figure 3

Prognostic analysis of selenoproteins in thyroid cancers using GEPIA platform. With a cut-off p-value of 0.05 , selenoproteins negatively correlated with pathological stages were shown in blue, selenoproteins positively correlated with pathological stages were shown in red. 


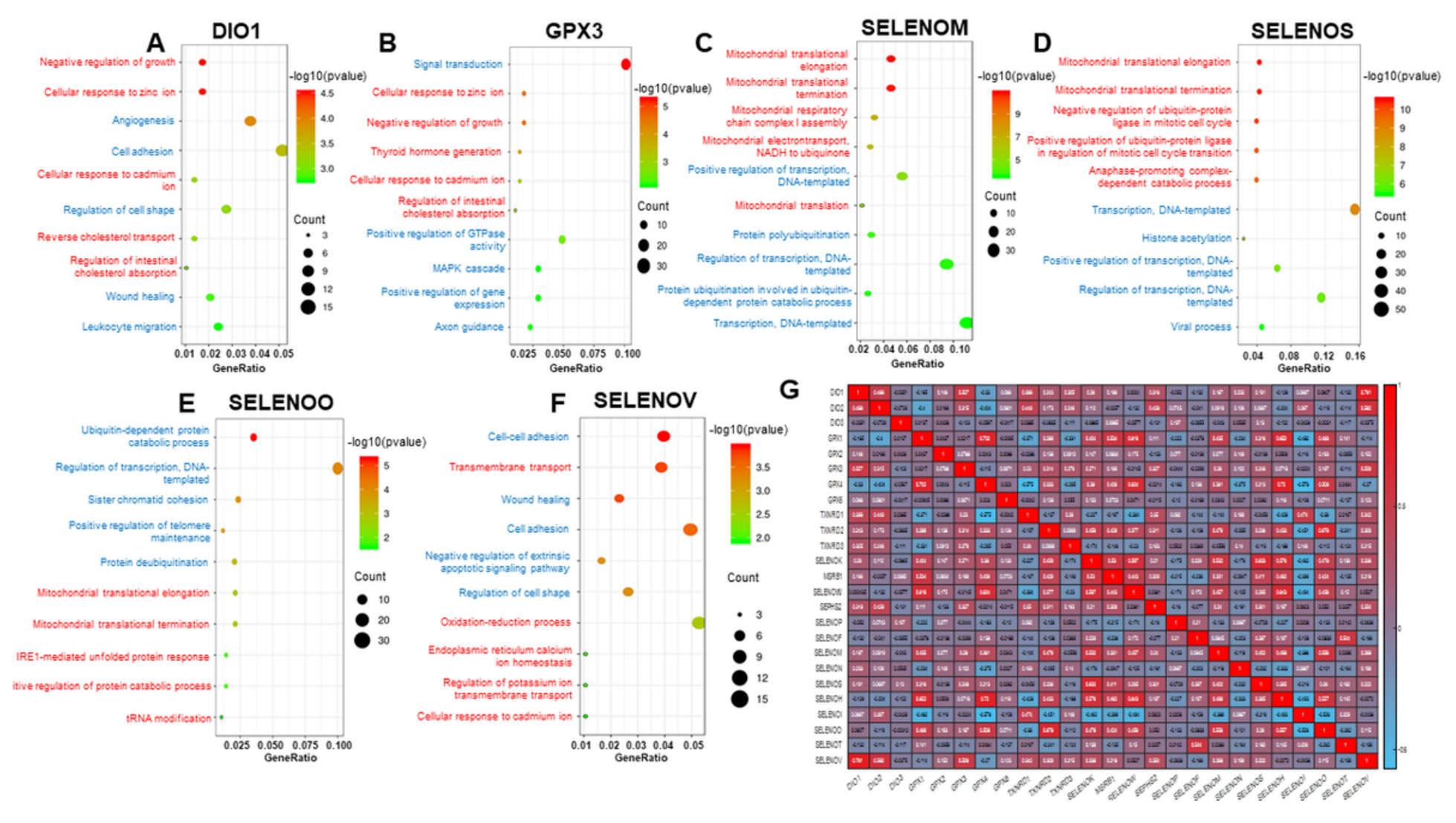

Figure 4

Gene Ontology (GO) and correlation analysis. (A-F) GO analysis of biological processes: the top 200 positive-correlated genes (red) and the top negative-correlated genes (blue) for DIO1 (A), GPX3 (B), SELENOM (C), SELENOS (D), SELEOO (E) and SELENOV (F) were analyzed. The top 5 biological processes that were significantly associated $(p<0.05)$ with the genes were plotted. $(G)$ Correlation analysis of selenoproteins with each other using cBioPortal online database. Correlations with $r \geq 0.6$ were shown in red.

\section{Supplementary Files}

This is a list of supplementary files associated with this preprint. Click to download.

- FigureS1.pptx

- SupplementaryDataS1.txt

- SupplementaryDataS2.txt

- SupplementaryDataS3.txt

- SupplementaryDataS4.txt

- SupplementaryDataS5.txt

- SupplementaryDataS6.txt

- SupplementaryDataS7.txt 
- SupplementaryDataS8.txt

- SupplementaryDataS9.txt

- SupplementaryDataS10.txt

- SupplementaryDataS11.txt

- SupplementaryDataS12.txt

- SupplementaryDataS13.txt

- SupplementaryFigureLegends.docx 